\title{
Novel technique for injecting and extracting beams in a circular hadron accelerator without using septum magnets
}

\author{
Andrea Franchi* \\ ESRF, CS 40220, 38043 Grenoble Cedex 9, France \\ Massimo Giovannozzi ${ }^{\dagger}$ \\ CERN, CH 1211 Geneva 23, Switzerland \\ (Received 8 January 2015; published 2 July 2015)
}

\begin{abstract}
With a few exceptions, all on-axis injection and extraction schemes implemented in circular particle accelerators, synchrotrons, and storage rings, make use of magnetic and electrostatic septa with systems of slow-pulsing dipoles acting on tens of thousands of turns and fast-pulsing dipoles on just a few. The dipoles create a closed orbit deformation around the septa, usually referred to as an orbit bump. A new approach is presented which obviates the need for the septum deflectors. Fast-pulsing elements are still required, but their strength can be minimized by choosing appropriate local accelerator optics. This technique should increase the beam clearance and reduce the usually high radiation levels found around the septa and also reduce the machine impedance introduced by the fast-pulsing dipoles. The basis of the technique is the creation of stable islands around stable fixed points in horizontal phase space. The trajectories of these islands may then be adjusted to match the position and angle of the incoming or outgoing beam.
\end{abstract}

DOI: 10.1103/PhysRevSTAB.18.074001

PACS numbers: 05.45.-a, 29.20.-c, 29.27.Ac, 29.27.Bd

\section{INTRODUCTION}

The injection of charged particle beams in most modern synchrotrons and storage rings, simply denoted hereafter as accelerators, from an upstream injector device is based on the combined use of a septum deflector and a system of pulsing dipoles (bumpers and kickers). Both are necessary since the nominal circular orbit defined by the ring main bending magnets cannot match that of the incoming beam [1].

Bumpers and kickers generate an orbit closed bump such that, at the septum exit, both the position and the angle of the bump match those of the incoming beam. In the first turn the kickers are quickly switched off (single-turn injection) or gradually reduced (multiturn injection) in order to prevent the injected beam from meeting the septum on the next turn. Once the injection process is completed, the bumpers are slowly turned off so as to bring the beam onto the nominal circular ring orbit without any effect on the transverse beam emittance. A typical injection scheme is sketched in Fig. 1. Basically, all on-axis injections of hadron beams into circular accelerators are based on this scheme, the only exception being that of the charge-exchange $\mathrm{H}^{-}$injection $[2,3]$.

\footnotetext{
*andrea.franchi@esrf.fr

†massimo.giovannozzi@cern.ch
}

Published by the American Physical Society under the terms of the Creative Commons Attribution 3.0 License. Further distribution of this work must maintain attribution to the author $(s)$ and the published article's title, journal citation, and DOI.
Lepton machines usually feature a slightly different approach in order to inject particles on top of a circulating beam (top-up mode), without the orbit bump crossing the septum blade. This results in the injected beam temporarily undergoing betatron oscillations. Due to radiation damping the new beam will naturally converge onto the nominal orbit preserving the natural emittance of the stored beam. This special operational mode increases the integrated luminosity in colliders, see, e.g., Ref. [4] and references therein, and photon flux in synchrotron light sources, while

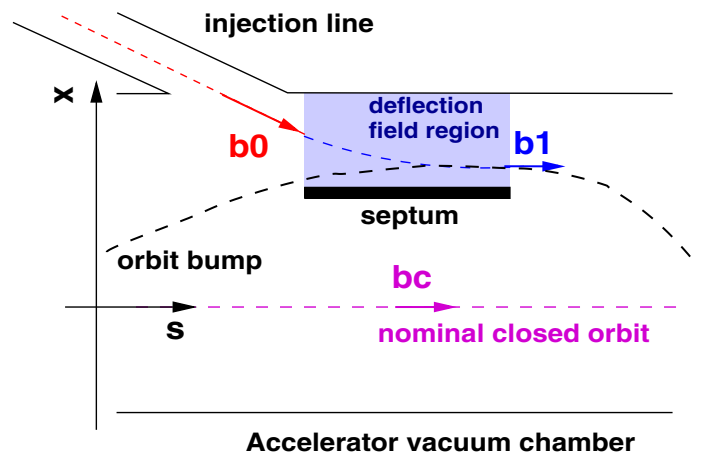

FIG. 1. Schematic picture of the standard on-axis injection by means of a septum and an orbit bump. At the injection into the ring, the beam initial conditions ( $b 0$ in the picture) are modified by the magnetic (or electrostatic) field screened to the rest of the accelerator by a septum. At its exit the beam conditions ( $b 1$ in the picture) are such to match the ones of the orbit bump created by kicker and bumper magnets. After the first turn the orbit bump is reduced until the closed orbit eventually returns to the nominal circular one ( $b c$ in the picture). 
reducing the thermal drifts in the experimental subsystems, hence increasing the overall operational efficiency. This type of off-axis injection is beyond the scope of this paper and will be dealt with in a separate article.

The fast (i.e., over one turn) extraction of beam toward either an experimental target or a downstream accelerator is performed by reversing the on-axis injection.

Magnetic septa are deployed to impart the largest deflection angle, typically of the order of tens of mrad, while kickers and electrostatic septa usually deviate the beam by only a few mrad. These values may vary according to the beam energy, accelerator size, and geometry of the injection and extraction regions. In principle, septa are not needed, provided that strong kickers, capable of imparting tens of mrad of deflection to the beam, can be designed, built, installed, and operated reliably. In practice the contrary is true: indeed, it is desirable to minimize the required kicker strengths by means of septa and bumpers in order to (mainly, though not only) reduce the impedance of the accelerator. Nevertheless, to maximize the deflecting field, septa usually induce aperture restrictions (vertical or horizontal for magnetic or electrostatic septa, respectively). Diffusive effects (such as scattering and space charge) displace particles at large transverse positions. Even in machines with sufficient dynamic aperture these particles may be eventually lost when hitting a physical restriction of the vacuum chamber, which in most cases is represented by septum magnets. Their removal would then increase the beam clearance, lower the losses of the stored beam during the entire accelerator cycle, reduce radiation on the equipment and, therefore, ease the machine hands-on maintenance.

It is well known that in the presence of magnets generating nonlinear fields, stable fixed points and islands can be generated. The essence of the novel method proposed in this paper is the observation that stable fixed points (and islands) represent alternative closed orbits (and stable regions) with respect to the standard case of the closed orbit at the origin of phase space. Moreover, the fact that these special closed orbits are located at a nonzero distance from the origin makes it possible to act on their evolution along the circumference using the ring optics, i.e., quadrupole magnets. Therefore, two sets of closed orbits are available in a circular accelerator, namely the standard orbit and those linked with the stable fixed points. Moreover, these two classes of orbits can be manipulated independently of each other. This opens the possibility of beam injection and extraction to be performed using these additional closed orbits, without septum magnets. This proposal goes in the direction of making particle accelerators more and more nonlinear, as far as beam dynamics is concerned, to exploit the richness of the behavior of nonlinear beam dynamics.

At this point we would like to draw the attention of the reader to the difference between the proposed method and recently refined injection methods based on pulsed magnets in synchrotron light sources (see, e.g., Refs. [5-8] and references therein). Indeed, these methods are based on the property that nonlinear magnets do not affect the orbit and optics of a circulating beam, while they can be used to act on injected beams which feature nonzero orbit excursions. No scheme based on pulsed sextupoles uses stable fixed points and stable islands, and these are the key elements of the novel approaches presented in this paper.

The layout of the paper is as follows: in Sec. II the proposed scheme is presented, starting from some general considerations about lattices and mathematical background reported in Secs. II A and II B, followed by the principles of the proposed scheme in Sec. IIC. The various types of extractions and injections based on these principles are discussed in Secs. III (multiturn processes), IV (singleturn extraction), and $\mathrm{V}$ (single-turn injection), while the discussion of specific points can be found in Sec. VI. Finally, the conclusions are presented in Sec. VII.

\section{THE PRINCIPLE OF THE PROPOSED SCHEMES}

\section{A. General lattice considerations}

The interest, i.e., the potential gain in terms of accelerator performance, and the feasibility of the proposed extraction schemes are highly dependent on the machine layout and optics. The presence in the ring layout of insertions dedicated to injection and extraction will certainly maximize the performance gain of these novel schemes. Indeed, a dedicated insertion allows the matching of the dispersion function and its derivative to zero, with a positive impact on the coupling between the longitudinal and transverse beam dynamics. Although this precondition is not strictly needed for the proposed schemes to work effectively, it certainly helps. Moreover, dedicated insertions would ensure the needed orbit deformations would cover fractions of the ring circumference in which no nonlinear magnets are located. Once more, this precondition is not mandatory, though it largely simplifies not only the implementation, but also the operation of these injection and extractions as it cancels the feed-down effect from magnets of higher order, which could even be timedependent when these orbit deformations are variable. Finally, a dedicated insertion would reduce the kicker strengths needed to the proposed schemes (see later).

The aim of this paper is to show the principles rather than one detailed implementation study, which would be based on a concrete design with specific performance goals. For this reason the lattice of the CERN Proton Synchrotron (PS) has been chosen to provide examples of the proposed extraction and injection schemes (the interested reader can find details of the PS lattice in, e.g., Refs. $[9,10]$ and references therein). It is clear that the PS lattice certainly does not feature the prerequisites mentioned above, such as dedicated insertions, zero dispersion in the straight 
sections, and bumps covering sections of the ring circumference which do not include nonlinear magnets. The basis for this decision is twofold: first, if the proposed schemes are shown to work on paper with a nonoptimal lattice, their performance will be even better with a more suitable lattice; second, the experience gained during the design of the multiturn extraction (MTE) [11] has provided tools for the analysis the PS lattice in the presence of stable islands related with low-order resonances. It should be noted that optics and tracking simulations presented in this paper have been carried out with the PTC modules [12-14] implemented in the MAD-X code $[15,16]$. Moreover, it is important to stress that the nonlinear magnetic fields unavoidably induced by the additional coil windings in the main magnets [10] add a non-negligible complication for the proposed extraction or injection schemes. Thus they have been artificially removed from the simulations presented here.

Another point should be clarified in order to avoid confusion. The ideas behind the proposals described in this paper are a spin off of the extraction based on the resonant beam splitting in stable islands of horizontal phase space [17,18], which has been implemented [11] and is being commissioned at the CERN PS [19-21]. Its key point was to split the beam in the horizontal plane when crossing a stable resonance, hence generating several beamlets that can then be extracted over more than one turn. This beam manipulation was named multi-turn extraction (MTE) by focusing on the extraction mode, which is performed using, among other devices, a septum magnet. The real novelty of that process, however, was indeed the splitting process. Throughout this paper the same term MTE will be used to indicate the same process with the novelty of removing the septum for the final extraction. Hence, it should be clear that the current proposal of a MTE extraction scheme is indeed original and independent, even if connected, to that implemented in the PS.

Parenthetically, a time-reversed process of beam merging has been proposed in view of performing a resonant multiturn injection (MTI) [22]. In this case, the injection of beam around the origin can be used as a means to shape the transverse beam distribution so as to mitigate space charge effects and preliminary studies have indeed confirmed that the shaping of the beam distribution survives the impact of space-charge effects [23].

\section{B. General mathematical considerations}

In a purely linear lattice the horizontal Poincaré section, i.e., the phase space portrait at a given location in the accelerator, is an ellipse, whose area $2 J \pi$ is an integral of motion. A symplectic transformation $(x, p) \rightarrow(\tilde{x}, \tilde{p})$ exists that maps the ellipse into a circle of radius $\sqrt{2 J}=|z|$, where $z(s)=\tilde{x}(s)+i \tilde{p}(s)=\sqrt{2 J} e^{-i \phi_{x}(s)}$. $\phi_{x}$ is the betatron linear phase, $2 J$ is an invariant quantity, $(\tilde{x}, \tilde{p})$ are referred to as Courant-Snyder (C-S) coordinates [24], and $(J, \phi)$ are action-angle variables. In the latter coordinates the corresponding Hamiltonian is $H=2 \pi Q_{x} J$, where the linear tune [25] $Q_{x}$ represents the natural oscillation frequency of the system.

By introducing magnets generating nonlinear fields, such as sextupoles and octupoles, the oscillation frequency depends on the action $J$ (amplitude-dependent detuning). The motion in the horizontal plane becomes quasiperiodic with the nonlinear tune $\nu_{x}$ [25] as main harmonic and secondary harmonics whose amplitudes depend on the strength of the nonlinear magnets [26,27]. At large amplitudes, where detuning and secondary harmonics are non-negligible, the phase space orbit is no longer an ellipse. Hence, it will no longer be mapped onto a circle by a C-S transformation. Its generalization to the nonlinear case is referred to as normal forms coordinates [28] $\xi(s)=\sqrt{\rho} e^{-i \theta(s)}$. In normal forms the phase space portrait is still foliated in circles of radius $\sqrt{\rho}=|\xi|$. Provided that $\rho$ is far enough from separatrices, it is the new invariant quantity under the effect of the nonlinear dynamics. In action-angle variables $(\rho, \theta)$ the 1D Hamiltonian reads

$$
H=2 \pi Q_{x} \rho-\frac{\Omega_{2}}{2} \rho^{2}-R(\theta) \rho^{2}+O\left(\rho^{3}\right),
$$

where $\Omega_{2}$ is the amplitude-dependent detuning coefficient and $R(\theta)$ is a resonant term [29] (not discussed here). The nonlinear tune $\nu_{x}$ can be written as

$$
\nu_{x}(\rho)=\frac{1}{2 \pi}\left\langle\frac{\partial H}{\partial \rho}\right\rangle_{\theta}=Q_{x}-\frac{\Omega_{2}}{2 \pi} \rho-\frac{\langle R\rangle_{\theta}}{\pi} \rho,
$$

where \langle\rangle$_{\theta}$ denotes the average over the angle variable. The detuning term $\Omega_{2}$ reads [29]

$$
\Omega_{2}=-\frac{1}{16} \sum_{l} K_{3, l} \beta_{x, l}^{2}+F\left(\beta_{x}, \phi_{x}, K_{2}\right),
$$

where the first sum extends over the number of octupoles, and $\beta_{x}, \phi_{x}$ are the horizontal C-S parameters [24]. The strengths $K_{3}$ and $K_{2}$ are the integrated magnetic strengths of octupoles and sextupoles, respectively, defined according to $K_{n}=l Z e / p\left(\partial^{n} B_{y} / \partial x^{n}\right)$, where $l$ represents the length of the magnet, $Z$ the charge state, $p$ the particle's momentum, and $B_{y}$ the vertical component of the magnetic field.

The function $F$ contains the contribution of sextupoles and is represented by a polynomial of order two, in which the second order monomials are of the form $K_{2, i} K_{2, j}$ with $i, j$ covering all sextupolar elements. The coefficients are complex expressions of the beta-functions at the locations $i$ and $j$ and of the phase advance between locations $i$ and $j$. The explicit form may be found in Ref. [29]. Control of $\Omega_{2}$ can be performed by using the strengths of octupole or sextupole magnets, noticing that $\Omega_{2}$ depends linearly on $K_{3, l}$. 
The assumption that the motion is governed by a 1D Hamiltonian is, of course, only an approximation, which can be made rather accurate if the nonlinear elements are placed at locations in which $\beta_{y} \ll \beta_{x}$, or when the beam emittances are different. Whenever, these conditions are not fulfilled, then the vertical degree of freedom needs to be included in these considerations and it can be shown [28] that the nonlinear tune is indeed described by the following expressions:

$$
\begin{aligned}
& \nu_{x}\left(\rho_{x}, \rho_{y}\right)=Q_{x}+h_{2,0} \rho_{x}+h_{1,1} \rho_{y}, \\
& \nu_{y}\left(\rho_{x}, \rho_{y}\right)=Q_{y}+h_{1,1} \rho_{x}+h_{0,2} \rho_{y},
\end{aligned}
$$

which clearly show a nonlinear coupling between the tunes in the horizontal and vertical planes. Equation (4) includes coefficients $h_{i, j}$, which describe the coupling between the two planes (indeed, $h_{2,0}$ is related with $\Omega_{2}$ ) and are functions of $K_{2}, K_{3}$. Hence, if the nonlinear coupling between horizontal and vertical planes is of concern, dedicated octupole magnets in regions with $\beta_{x} \simeq \beta_{y}$ can be used, as discussed in Ref. [21], to minimize or nullify the coefficient $h_{1,1}$.

The detuning with amplitude is fundamental to the formation and manipulation of stable islands, since it allows the existence of fixed points when the linear tune $Q_{x}$ is separated from the resonance condition $\bar{Q}_{x}$ by $\Delta=Q_{x}-\bar{Q}_{x}$. This is easily proved by searching in Eq. (2) a $\rho^{*}$ that, for a given linear tune $Q_{x}$, locks $\nu_{x}=\bar{Q}_{x}$, hence making those points always undergo the resonant tune [28]

$$
\rho^{*}\left(\Delta, \Omega_{2}\right)=-\frac{2 \pi \Delta}{\Omega_{2}+2\langle R\rangle_{\theta}} \simeq-\frac{2 \pi \Delta}{\Omega_{2}}+O\left(\Delta^{2}\right) .
$$

The reminder in the above equation includes the resonant term, which is proportional to $\Delta$ [29]. The weak linear dependence of $\Omega_{2}$ on $\Delta$, through $F$ and $\beta_{x}$, is neglected, but it can easily be included in the computations if necessary. The existence of fixed points is determined by the signs of both $\Delta$ and $\Omega_{2}$ : for a positive value of $\Omega_{2}$, fixed points exist only when $Q_{x}<\bar{Q}_{x}, \rho^{*}$ being always positive. In this paper $\Omega_{2} \neq 0$ is assumed. If this condition is not met, higherorder terms need to be included, as has been done in Ref. [29]. Once more, the principle proposed here is not affected by such detail. For any resonant tune $\bar{Q}_{x}=I+1 / q$, where $I$ is the tune integer part and $q$ the resonance order, the Poincaré-Birkhoff theorem [30-32] ensures that $q$ fixed points exist at amplitude $\sqrt{\rho^{*}}$ and phases $\theta(s)+2 n \pi / q, 1 \leq n \leq q$, where $\theta(s)$ has the same evolution as $\phi_{x}(s)$. Resonant tunes of the form $\bar{Q}_{x}=I+m / q$, with $m$ integer and $m<q$, would generate the same phase space topology with $q$ fixed points. The only difference for a beam trapped around one would be in the sequence to which it moves onto the others
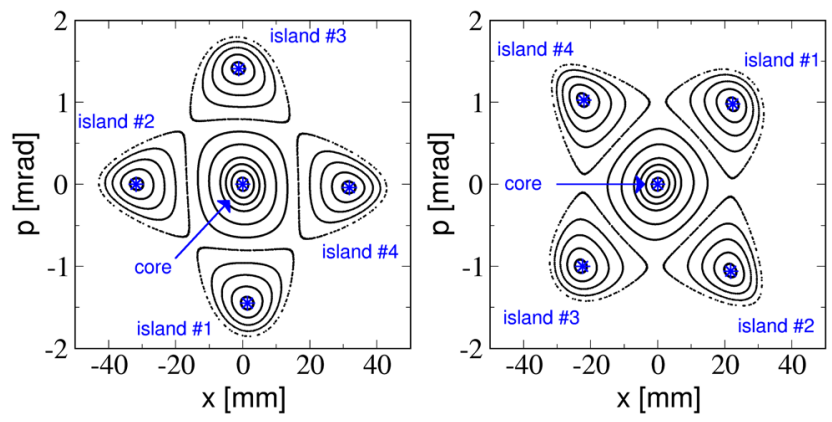

FIG. 2. Examples of horizontal phase space portraits at two different locations of the CERN PS ring in the presence of stable islands of fourth-order resonance. Blue stars denote the fixed points, which rotate in phase along the ring as in Fig. 3.

turn-by-turn. In the case of the one-fourth $(q=4)$ resonance of Fig. 2 and $m=1$ a beam trapped into the island \#1 (at a given section along the ring) would occupy the island \#2 after one turn, the \#3 after two turns, the \#4 after three turns before coming back to the island \#1 after four turns. With $m=3$ the occupancy sequence would be: \#1, \#4, \#3 and \#2.

The last missing ingredient to really illustrate the idea behind the proposed scheme is the evaluation of the fixed point coordinates in the Cartesian frame $(x, p)$. It is worth noting that all coordinate transformations used so far, i.e., $\mathrm{C}-\mathrm{S}$ and normal forms, are always chosen to be tangent to the identity, implying that $\sqrt{\rho} e^{-i \theta}=\xi \simeq z+O(\rho)$. This results in

$$
\begin{gathered}
\left(\begin{array}{c}
\tilde{x} \\
\tilde{p}_{x}
\end{array}\right)=\left(\begin{array}{c}
\sqrt{2 J} \cos \phi_{x} \\
-\sqrt{2 J} \sin \phi_{x}
\end{array}\right) \simeq\left(\begin{array}{r}
\sqrt{\rho} \cos \theta \\
-\sqrt{\rho} \sin \theta
\end{array}\right), \\
\left(\begin{array}{c}
x \\
p_{x}
\end{array}\right)=\left(\begin{array}{cc}
\sqrt{\beta_{x}} & 0 \\
-\alpha_{x} / \sqrt{\beta_{x}} & 1 / \sqrt{\beta_{x}}
\end{array}\right)\left(\begin{array}{c}
\tilde{x} \\
\tilde{p}_{x}
\end{array}\right) .
\end{gathered}
$$

After merging the above equations and evaluating them for the fixed points $\left(\rho^{*}, \theta^{*}\right)$, the corresponding Cartesian coordinates read

$$
\begin{aligned}
& x^{*}\left(\Delta, \Omega_{2}, s\right) \simeq \sqrt{\beta_{x}(s) \rho^{*}\left(\Delta, \Omega_{2}\right)} \cos \theta^{*}(s) \\
& p^{*}\left(\Delta, \Omega_{2}, s\right) \simeq-\sqrt{\frac{\rho^{*}\left(\Delta, \Omega_{2}\right)}{\beta_{x}(s)}} \\
& \times\left[\alpha_{x}(s) \cos \theta^{*}(s)+\sin \theta^{*}(s)\right] \\
& \theta^{*}(s) \simeq \phi_{x}(s)+\frac{2 n \pi}{q}, \quad 1 \leq n \leq q .
\end{aligned}
$$

The phases of the $q$ fixed points depend on the local betatron phase $\phi_{x}(s)$ and are separated by $2 q / \pi, q$ being the order of the resonance. Their position and angle $\left(x^{*}, p^{*}\right)$ depend on the invariant $\rho^{*}$ of Eq. (5) and the local C-S 
parameters $\beta_{x}(s)$ and $\alpha_{x}(s)$. The orbit of the $q$ fixed points may then be manipulated either by varying nonlinear magnets (i.e., $\Omega_{2}$ ) or by changing the linear tune (i.e., $\Delta$ ) or by a local modification of the linear optics [i.e., $\beta_{x}(s)$ and $\alpha_{x}(s)$ ].

\section{Outline of the proposed approach}

As already mentioned, the idea of the proposed scheme is to use the fixed points inside stable islands with the aim of creating additional closed orbits in a circular accelerator. More quantitatively, Eqs. (8)-(9) are the core of the proposed scheme. Indeed, they show that, by using nonlinear magnets, it is possible to create stable fixed points in the horizontal phase space. This, in turn, generates a closed orbit that winds up along the accelerator circumference and closes after $q$ turns, hence providing $q$ channels available for injection or extraction. The existence of $q$ channels is related to the fact that $q$ fixed points exist at a certain distance from the origin (proportional to $\sqrt{\rho^{*}}$ ) with $q$ different phases $\theta^{*}$, as shown in Eq. (9). After $q$ turns the phases return to their initial values, hence closing the orbit. The second closed orbit is off-center and will be affected by quadrupole magnets through their feed-down fields and the local C-S parameters $\beta_{x}, \alpha_{x}$, and $\phi_{x}$. Therefore, an appropriate choice of the optics in a dedicated section of the accelerator, together with a proper setting of the tune, i.e., of $\Delta$, and of the octupole strength, i.e., $\Omega_{2}$, makes possible the matching between one of the $q$ conditions $\left(x^{*}, p^{*}\right)$ and that of the incoming (or outgoing) beam without need of a septum magnet. This can only be feasible if two conditions are met in the design of the linear optics of the dedicated section: (i) The injection (or extraction) point must be located outside magnetic elements, i.e., in an empty straight section; (ii) $p^{*}$ must equal the angle of the incoming (or outgoing) beam.

\section{MULTITURN EXTRACTION AND INJECTION}

To perform a multiturn extraction (MTE) the first step consists of preparing the beam for this special extraction mode. This can be achieved by crossing a stable resonance slowly. It is worth recalling that the number of extracted turns is linked to the order of the resonance used and to its stability type: If the resonance of order $q$ is stable, then $q+1$ turns will be extracted, whereas only $q$ turns will be extracted in the case of unstable resonance.

The transverse splitting is mastered at the CERN PS, where about $75 \%$ of the beam is moved into four islands (each accommodating one fourth of the total) and the remaining $25 \%$ is kept on the nominal zero orbit (the socalled core). Prior to extraction, the horizontal orbits of the five beamlets look like those of the top plot of Fig. 3, whereas phase space portraits at two different locations around the ring are displayed in Fig. 2. The extraction is
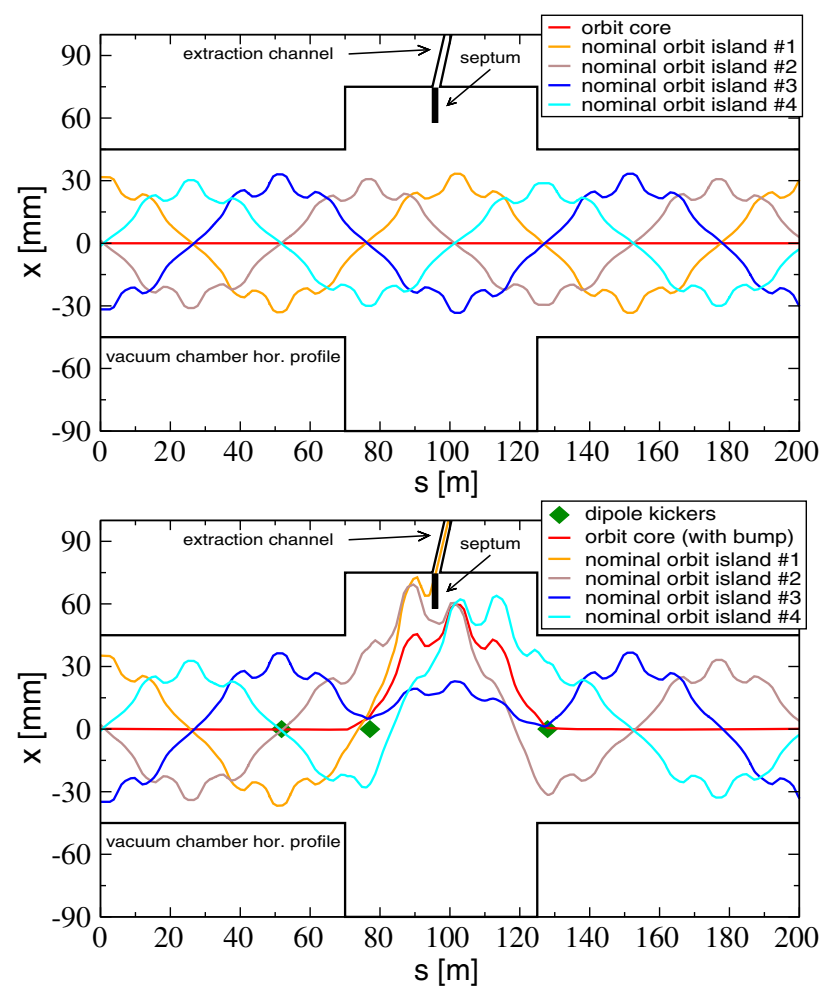

FIG. 3. Standard, with septum, MTE at the CERN PS. Top plot: the closed orbits for the four fixed points (islands) and the nominal central ring orbit (core) prior extraction. Bottom plot: the same orbits during the fast orbit bump created by three dipole kickers: To be extracted, the beam trapped in the first island needs a deflection from the magnetic septum. Only a fraction of the PS circumference (200 $\pi \mathrm{m}$ long) is shown.

obtained by generating an orbit bump (with three dipole kickers) so as to have one fixed point only entering the septum magnet, as shown in the lower plot of Fig. 3.

Thanks to the nonzero position of the fixed points, the perturbation to their orbits can be achieved by using either a quadrupolar or higher-order magnetic field generating a dipolar force by feed down from its main field. The choice of the type of magnetic field greatly depends on the machine optics and the required magnetic strength. In principle, in order to extract the four islands, quadrupolar kicks are sufficient to modify the island's orbit quickly enough to make the first island enter the extraction channel. The quadrupolar perturbation shall be closed, to ensure that each of the remaining islands are extracted over the following turns. In Fig. 4 the perturbed orbits are displayed. The change of the insertion optics created by a set of quadrupoles distorts locally the orbits of all islands, while leaving unchanged orbits and optics outside the insertion. The beam in the first island enters the extraction channel during the first turn of MTE, while the remaining three keep circulating. Note that the orbit of the first island downstream of the extraction point, even if displayed, is actually empty, the beam being extracted and no longer 
following the magnetic forces present in the ring. On the next turn, the second island will occupy the same orbit as the first island during the previous turn and will be extracted. The insertion optics performed over four turns ensures the extraction of all islands without septum or dipolar kickers. As far as mechanical aperture is concerned, local modifications of the vacuum chambers may be needed to provide enough clearance depending on the machine layout.

In the case of Fig. 4 two pairs of quadrupole kickers (with integrated magnetic fields not greater than $5 \mathrm{~T}$ ) are used to preserve the tune and the full periodicity over the four turns. The actual number of quadrupoles and their strengths largely depends on the machine optics and parameters, so that Fig. 4 has to be considered only indicative. Clearly, the insertion optics has no impact on the central orbit, but further displaces (as done by the orbit bump) and deflects (as done by the septum) the islands.

The extraction of the beam core can be performed by providing a fast deflection using a dipole kicker to jump onto the same fixed point as used to extract the previous turns. This is equivalent to a fast extraction whose details are discussed in the next section. In conclusion, the whole set of $q+1$ beamlets can be extracted without the need of a septum magnet.
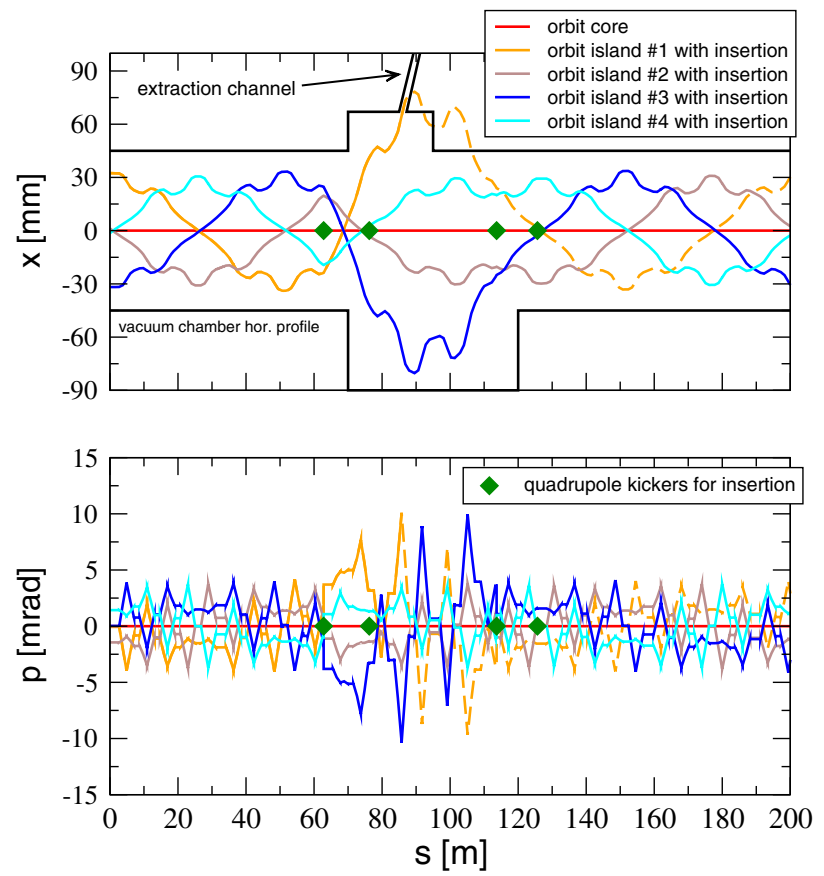

FIG. 4. Modified, septumless, MTE. The distortion of the four islands' closed orbits is induced by local insertion optics created by quadrupole kickers, thus leaving the central orbit unaffected. The insertion is optimized so as to have precisely one orbit entering the extraction channel and to ensure that, turn-by-turn, all other islands' orbits will exit with the same position and angle. Neither dipole kickers nor septa are used to achieve this result. Only a fraction of the PS circumference is shown.
The inverse procedure may be implemented for a resonant MTI. Two variants can be devised, depending on whether injection on the center of phase space is envisaged or not. In fact, whenever only injection into the islands is required, then dipolar kickers and septum are no longer needed, because no beam has to be injected directly into the core. After completion of the injection process, the beamlets will be moved toward the center. The merging of islands is achieved by crossing the same resonance ( $1 / 4$ in the case of the CERN PS) in the opposite direction with respect to the MTE.

The matching of the optical conditions between the transfer line and the receiving island at the injection point of the ring can be addressed in a straightforward way by computing the $\mathrm{C}-\mathrm{S}$ parameters around the receiving fixed point and to impose them on the optics of the transfer line. Of course, the same needs to be done for the dispersion and its derivative. Note that in general each fixed point has its own C-S and dispersion functions (hence different from those of the nominal on-axis orbit). They can be computed either directly by the PTC module of MAD-X or indirectly via single particle tracking close to the fixed points, by fitting the orbit with ellipses (for the C-S parameters) and by evaluating the fixed points' displacement against energy deviation (for the dispersion functions).

The detail of the merging process after MTI can be found in Ref. [22], where it was shown that, even in the presence of nonlinear forces during the whole merging process, the final beam emittance is still lower than that of a classical, i.e., without islands, multiturn injection. Furthermore, in Ref. [23] it was shown that the presence of space charge effects has a minor impact on the beam properties, thus showing that the merging process by means of stable islands is indeed robust even in the presence of space charge.

Finally, it should be noted that the longitudinal beam dynamics has time scales much longer, effectively decoupling transverse and longitudinal motions. No impact from the longitudinal dynamics is to be expected; neither at the level of MTI, which occurs over few turns, nor at the level of the beam merging process.

\section{SINGLE-TURN EXTRACTION}

Two options are available to perform a single-turn extraction (STE), also called fast extraction. The first consists of using one of the $q$ fixed points of a stable resonance of order $q$. Those islands are not populated with beam prior to extraction, the particles being still confined to the central orbit (the core). The machine setting (horizontal tune and nonlinear magnets), or a local insertion optics similar to that of Fig. 4, can be designed so as to have one of the $q$ fixed points matching the extraction conditions (position and angle). During the last turn prior to STE, at a waist $\left(d \beta_{x} / d s=0\right)$ upstream of the extraction point (see left picture of Fig. 2), a dipole kicker is fired to 
displace the beam circulating on axis onto the fixed point with $x^{*}=0$ and $p_{x}^{*}<0$ (corresponding to island \#1 in that figure). Thanks to the overall machine setting the displaced beam will enter the extraction line. Just upstream of the extraction point the accelerator will have no magnetic elements and the trajectory will be straight. Without an orbit bump, forcing a fixed point to move across a symmetric vacuum chamber, will result in having at least one other fixed point to do the same on the opposite side (see Fig. 4). However, this is of no concern, as the islands are not populated, until the last turn. Even when the dipolar kicker is fired, only the island that fits the extraction channel geometry will be populated, whereas the remaining $q-1$ islands will remain empty.

In Fig. 5 an example based on the one-fourth resonance, $Q_{x} \simeq 6.25$, at the CERN PS is shown. The dipolar kicker is located at a waist so as to displace the on-axis beam onto a fixed point trajectory. The latter is such that the beam enters the extraction channel with no need for an additional deflection imparted by a septum. Once there, the beam drifts away, abandoning the fixed point, as the accelerator magnetic forces are being screened by the channel itself.

The kicker strength required to displace the beam from the central orbit to one fixed point may be relatively large,

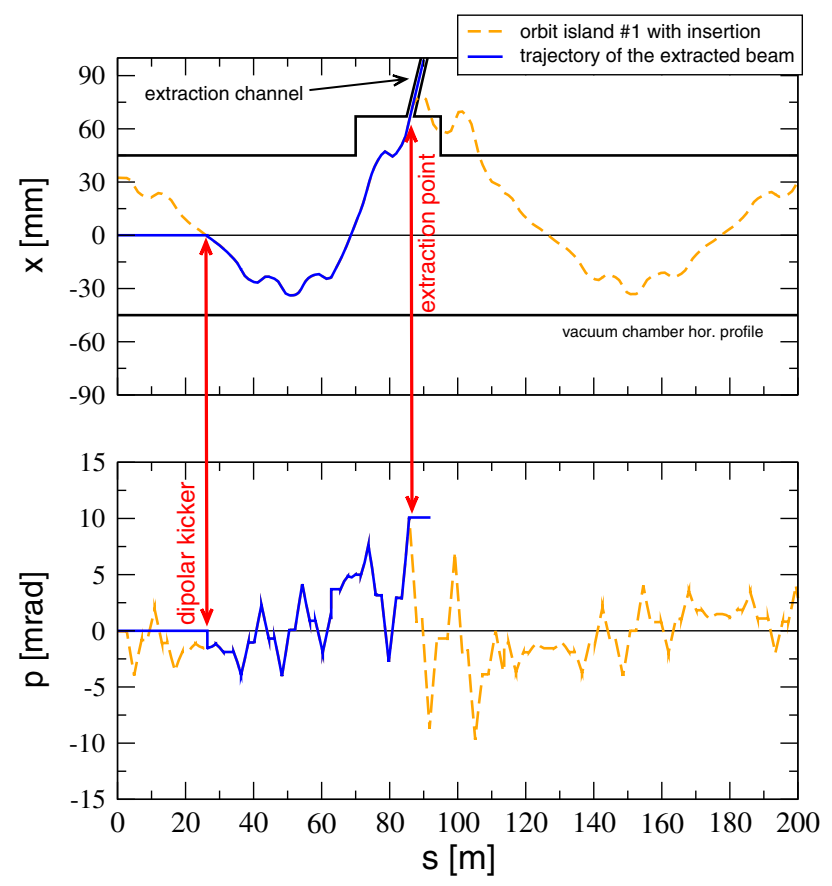

FIG. 5. Example of the beam trajectory (blue) during the last turn of an STE. At a horizontal waist the central beam is displaced onto a fixed point with $x^{*}=0$ and $p_{x}^{*}<0$ by a dipolar kicker. This point and the beam trapped in the corresponding island follow an orbit that matches the extraction conditions without having to use a septum to impart the necessary deflection. This example is based on the four-island MTE of the CERN PS: the orbits of the other 3 islands (already shown in Fig. 4) are not displayed as they are not populated. depending on the corresponding $p^{*}$ of Eq. (9). In the case of Fig. 5, about $1.5 \mathrm{mrad}$ of deflection would be necessary to reach the fixed point. Nevertheless, additional insertion optics around the dipolar kicker can be designed to make the fixed point approach the central orbit, hence reducing the kicker strength. The idea is to have an insertion featuring large $\beta$ values with very low divergence: According to Eqs. (8)-(9), such a waist $\left(\alpha_{x} \simeq 0\right.$ and $\cos \theta^{*}(s)=0$ ) would result in one fixed point with zero position and low divergence. In Fig. 6 an example is shown, with a second insertion created by two pairs of quadrupoles, each separated by a horizontal phase advance of $\pi$. This modification reduces the angle $p$ of island \#1, receiving the beam at the position of the dipole kicker, from 1.5 to $1 \mathrm{mrad}$, corresponding to a reduction of 33\% in the required strength. It goes without saying that the strength reduction is intimately connected with the properties of the ring lattice. Hence, one should not consider these values as absolute, but only as indications that appropriate optics is crucial to optimize the kicker's strength. Indeed, high-beta optics, as implemented in the CERN LHC for the TOTEM [33] and ATLAS/ALFA [34] Experiments [35] could further reduce the required strength of the kicker.

The second option for a septum-free STE consists of creating a second closed orbit by using a stable fixed point of period one, i.e., such that this additional closed orbit

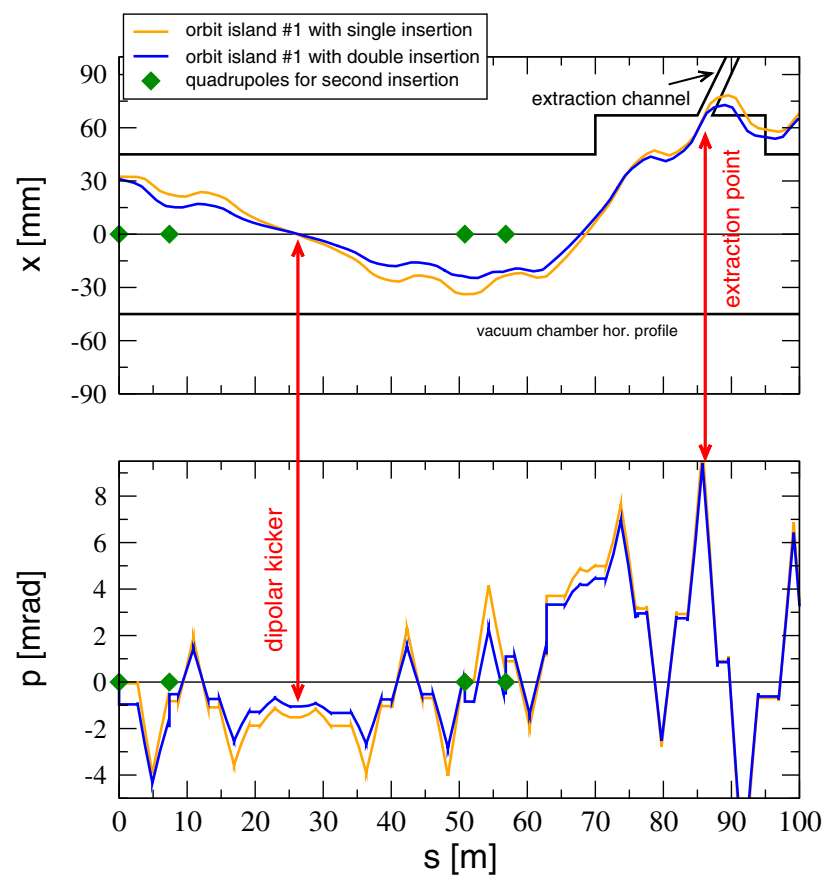

FIG. 6. Example of optics manipulations with four quadrupoles to reduce the strength of the dipole kicker necessary to displace the beam from the central orbit to the one used for performing STE. The initial island orbit with the same insertion optics of Fig. 5 (orange) requires a deflection of $1.5 \mathrm{mrad}$ from the dipole kickers, whereas only $1 \mathrm{mrad}$ would be needed with the proposed insertion. 
closes after one single machine turn. This special configuration can be achieved by using octupoles in conjunction with a rather low fractional part of the horizontal tune. In Fig. 7 an example is showed for the CERN PS lattice with $Q_{x}=6.05$ and one octupole powered so to have the stable fixed point matching the extraction condition (bottom right picture). As for the fast extraction with the stable fourthorder resonance, the beam circulating on the central orbit is displaced into the island at an upstream waist (bottom left picture). The large angle (about $5 \mathrm{mrad}$ in this example) can be reduced by dedicated insertion optics as in Fig. 6 .

The surface of the islands surrounding the stable fixed points decreases with the order of the resonance: the lower the order, the larger the area. The extraction of low-intensity beams with large horizontal emittance
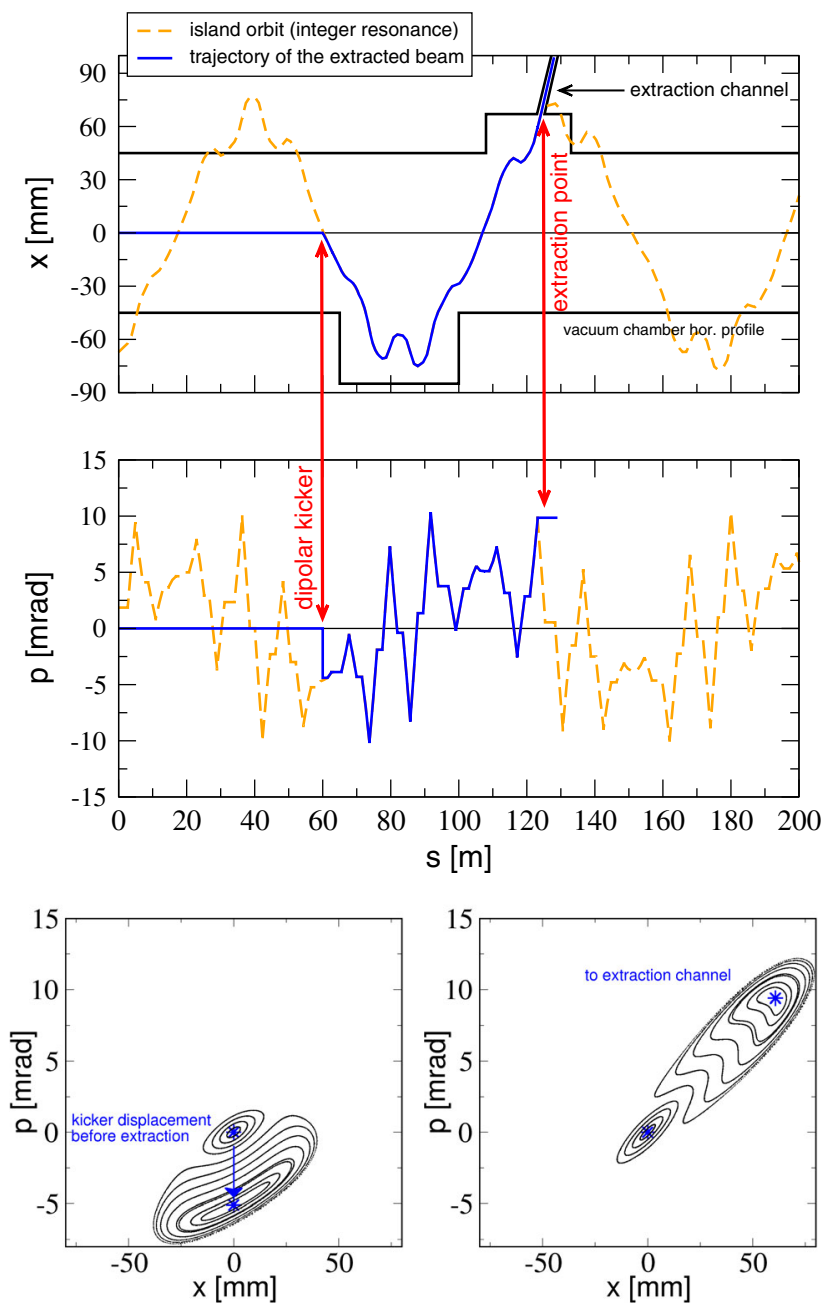

FIG. 7. Example of STE obtained by moving the beam from the central orbit to one inside a stable island of the integer resonance. The island orbit and the trajectory during extraction are displayed in the upper plots (orange and blue curves, respectively), whereas the phase space portraits in the bottom plots refer to the location of the dipolar kicker (left) and of the extraction point (right). The large deflection angle of about $5 \mathrm{mrad}$ can be reduced by appropriate insertion optics around the kicker as shown in Fig. 6. would then benefit from the integer resonance of Fig. 7. High-intensity beams with moderate or low emittance would instead be better extracted by operating close to resonances of order higher than two in order to keep a safe distance from the integer and half-integer resonances. Another consideration that could be made to select the order of the resonance used to perform STE is based on the vicinity of the resonant tune to the standard accelerator working point. This would reduce the tune change prior to extraction needed to create the islands, thus minimizing the potential risk of spoiling the beam quality due to tune sweeping across the resonance net.

It is important to comment on the special optics that might mitigate possible issues with the strength of the extraction kicker. Such optics, which could be implemented as dedicated insertion optics, could be used either during the whole machine cycle, i.e., from injection to extraction, or implemented only prior to the extraction stage. It must be emphasized that the latter scenario does not require any quadrupolar kicker, which is necessary for the MTE. In fact, slow pulsing quadrupoles can drive the optics transition from that used during store to that of the STE. Therefore, no matter whether a set of additional $q$ orbits or only one are used for STE, a fast dipolar kicker is needed plus, possibly, slow pulsing quadrupoles.

\section{SINGLE-TURN INJECTION}

The same technique may be reversed for a single-turn injection (STI), with a dedicated machine setting, namely horizontal tune and nonlinear magnets, generating one out of $q$ fixed points with the correct position and angle to match those of the beam coming from the injection line. A fast kicker can be placed downstream at a convenient position, i.e., at the waist, to displace the beam trapped in the island onto the standard circular ring orbit. As with the STE, machine setting and kicker position can be optimized, together with the insertion optics, to minimize the kicker strength. After injection, the octupole(s) used to generate the stable fixed point(s) can be turned off, making the stable islands disappear (when $\Omega_{2} \rightarrow 0, \rho^{*} \rightarrow \infty$ ). This stage is supposed to preserve the injected beam quality, as long as the time-variation of the octupole strength is slow enough to be adiabatic.

The matching of the optical conditions between the transfer line and the receiving island at the injection point of the ring can be solved as already discussed for MTI. This is however not enough to prevent filamentation induced by optical mismatch when displacing the incoming beam trapped in the island onto the nominal circular orbit. Since this shift occurs at a waist (betatronic and dispersive) a dedicated insertion needs to be designed so to have the fixed points' $\beta$ and dispersion functions to match as closely as possible those of the standard closed orbit. In order to prevent any possible emittance growth or filamentation between injection point and kicker, the surface of the stable 
island has to be large enough to accommodate the whole beam, to prevent particles from crossing separatrices in phase space.

As a last point, it is worth mentioning that the longitudinal beam dynamics, e.g., rf capture, cannot affect the STI process (and vice versa) as the time scales are completely different.

\section{DISCUSSION OF THE PROPOSED APPROACH}

It can be argued that for MTE, MTI or STE, STI the ring can be better represented as a transfer line, hence questioning the usefulness of the approach proposed here, which is based on periodic structures. For the case of MTE and MTI it is easy to justify the use of periodic structures to avoid beam filamentation, due to the beam dynamics at large amplitude, over the injection or extraction processes. On the other hand the use of fixed points for STE or STI is questionable. In fact, a proper displacement can be found such that the resulting trajectory matches the extraction conditions thanks to suitable dipolar feed-down fields of the magnets between the dipole kicker and the extraction point (i.e., the insertion optics). This is undoubtedly true. However, it is our opinion that dealing with closed orbits of stable islands, rather than plain trajectories, is of great help designing, characterizing, commissioning, and operating these septum-free STE or STI. Moreover, the presence of stable fixed points with islands around them ensures that the emittance growth during the extraction or injection process is smaller than when without these stable structures of phase space. This is because the phase space dilution caused by the geometric detuning with standard optics and experienced by the beam moving at large amplitudes is greatly reduced when the beam is put onto a stable fixed point, whose tune is locked by definition, see Ref. [11].

The special phase space topology required for performing the proposed septumless injection or extraction can be generated either statically or dynamically.

The static configuration (i.e., constant $\Delta, \Omega_{2}, \rho^{*}$ and hence steady fixed points' position) implies that the tune is set close to the resonant tune and the nonlinear elements are set to generate the stable islands with strengths that are time-independent. This, of course, will limit the choice of the machine tune, which might be a too strong constraint in terms of flexibility and operability of the ring. The only degree of freedom might be the choice of the fractional part of the tune. The impact of the nonlinear elements is of much less concern. Provided that the stable region around the central orbit (the core of Fig. 2) is large enough to contain the beam, the presence of islands beyond its contour is of no concern as far as both the stability and the properties of the beam are concerned.

The dynamic configuration (i.e., $\Delta, \Omega_{2}, \rho^{*}$ and hence fixed points' position variable in time) is, of course, the most flexible as it envisages the change of both working point and strength of the nonlinear elements with time so that the required phase space topology would be generated only when needed. The change of the fractional part of the tune is to be considered with care as resonance crossing might impact beam quality: So this option should be assessed on a case-by-case basis. Nonetheless, in most colliders the working point is safely, in terms of beam properties, changed dynamically before putting beams in collisions (see Ref. [35] for more detail). Therefore, it seems not too restrictive to assume that the tune of the ring can be chosen freely apart from the injection or extraction stages. As experience with the standard MTE at the CERN PS has demonstrated [21], an adiabatic (i.e., slow) dynamical change of the strength of the nonlinear elements has no impact on the beam quality.

Depending on the machine layout, the large orbit excursions of the beam trapped into the islands may require a local modification of the vacuum chambers in order to ensure sufficient physical aperture, as shown in Figs. 3-7. In the case of the standard MTE implemented at the CERN PS, some vessels needed to be replaced by structures with larger horizontal aperture downstream the extraction septum [11]. Because of the PS combined-function bending magnets, there was no need of quadrupole magnets with large apertures. This may not be the case for machines with separated-function magnets.

The proposed extraction and injection schemes require not only fast dipoles, but also fast quadrupoles. While fast dipoles are standard systems in modern accelerators, albeit sometimes challenging in terms of technology, higher-order fast magnets are less common. As already mentioned, the aim of this discussion is not a detailed analysis of the strength requirement of the elements that are part of the current proposal. Nevertheless, at least at the level of principle, it is possible to state that no fundamental showstopper is in sight for the development of fast magnets of quadrupolar type or higher order. Indeed, Ref. [7] reports the successful operational use of fast sextupoles at the photon factory storage ring in KEK, thus supporting that, even if challenging, designing and building a quadrupolar kicker is not a prohibitive task.

\section{CONCLUSIONS}

In this paper a novel approach to perform injection into and extraction from a circular particle accelerator without septum magnets has been presented. It relies on the generation of stable fixed points and islands in the horizontal phase space via the use of normal nonlinear magnetic elements, such as sextupoles and octupoles (their skew counterparts may be deployed to perform the same manipulation in the vertical phase space). These techniques have been illustrated using the lattice of the CERN PS ring. Such a lattice is certainly not particularly suited to this configuration and the results presented here should be 
considered only as examples of the feasibility of the proposed approach.

The off-axis trajectories of the fixed points represent additional closed orbits with respect to the standard on-axis orbit. The betatronic amplitude of these additional orbits can be controlled using the linear machine tune and the strength of the nonlinear elements. Moreover, being at nonzero amplitude, the feed-down effects from quadrupoles or higher-order magnets can be used to shape these orbits to match the injection or extraction conditions.

These considerations can be applied to both single-turn as well as multi-turn processes. In both cases the septum magnets can be replaced by an appropriate design of the optics of the ring. Closed, slow bumps can still be used to optimize the conditions required to perform injection or extraction from the ring.

Single-turn processes require the use of a single fastpulsing dipole, whereas multiturn processes require the use of at least two fast-pulsing magnets generating a field of quadrupolar type or higher order.

\section{ACKNOWLEDGMENTS}

We are grateful to $\mathrm{R}$. Tomás for proofreading the original manuscript and making valuable suggestions. We would like to express our warm thank to E. McIntosh for reading and improving greatly this manuscript. We would also like to express our gratitude to the two referees for the excellent and stimulating comments.

[1] A. W. Chao and M. Tigner, Handbook of Accelerator Physics and Engineering (World Scientific, Singapore, 1999), ISBN 9810235003.

[2] G. I. Budker and G. I. Dimov, On the charge exchange injection of protons into ring accelerators, in International Conference on High-Energy Accelerators, Dubna (AEC, Oak Ridge, TN, 1963), p. 1372, http://babel.hathitrust.org/ cgi/pt?id=mdp.39015086509943; view=1up;seq=682.

[3] C. W. Potts, Negative hydrogen injection into the zero gradient synchrotron, IEEE Trans. Nucl. Sci., 24, 1385 (1977).

[4] U. Wienands, Lepton collider operation with constant currents, in Proceedings of the 21st Particle Accelerator Conference, Knoxville, TN, 2005, edited by C. Horak (IEEE, Piscataway, NJ, 2005), p. 149, http://accelconf .web.cern.ch/AccelConf/p05/PAPERS/WOAD002.PDF.

[5] K. Harada, Y. Kobayashi, T. Miyajima, and S. Nagahashi, PF-AR injection system with pulsed quadrupole magnet, in Third Asian Particle Accelerator Conference, edited by W. Namkung and S. Baik (Pohang Accelerator Laboratory, Gyeongju, 2004), p. 344, http://accelconf.web.cern.ch/ AccelConf/a04/PAPERS/TUP14001.PDF.

[6] K. Harada, Y. Kobayashi, T. Miyajima, and S. Nagahashi, New injection scheme using a pulsed quadrupole magnet in electron storage rings, Phys. Rev. ST Accel. Beams 10, 123501 (2007).
[7] H. Takaki, N. Nakamura, Y. Kobayashi, K. Harada, T. Miyajima, A. Ueda, S. Nagahashi, M. Shimada, T. Obina, and T. Honda, Beam injection with a pulsed sextupole magnet in an electron storage ring, Phys. Rev. ST Accel. Beams 13, 020705 (2010).

[8] S. C. Leemann, Pulsed sextupole injection for Sweden's new light source MAX IV, Phys. Rev. ST Accel. Beams 15, 050705 (2012).

[9] A. Franchi, S. Gilardoni, and M. Giovannozzi, Progresses in the studies of adiabatic splitting of charged particle beams by crossing nonlinear resonances, Phys. Rev. ST Accel. Beams 12, 014001 (2009).

[10] S. Gilardoni and D. Manglunki et al., Fifty years of the CERN proton synchrotron, Report No. CERN-2011-004, 2011, https:// irradiation.web.cern.ch/irradiation/Paper/cern2011-004.pdf.

[11] M. Giovannozzi et al., The CERN PS multi-turn extraction based on beam splitting in stable islands of transverse phase space, Report No. CERN-2006-011, 2006, https:// cds.cern.ch/record/987493/files/CERN-2006-011.pdf.

[12] F. Schmidt, E. Forest, and E. McIntosh, Introduction to the polymorphic tracking code, Report No. CERN-SL-2002044-AP, 2002, https://cds.cern.ch/record/573082/files/ sl-2002-044.pdf.

[13] F. Schmidt, MAD-X PTC integration, in Proceedings of the 21st Particle Accelerator Conference, Knoxville, TN, 2005, edited by C. Horak (IEEE, Piscataway, NJ, 2005), p. 1272, http://accelconf.web.cern.ch/AccelConf/p05/ PAPERS/MPPE012.PDF.

[14] P. K. Skowronski, F. Schmidt, and E. Forest, Advances in MAD-X using PTC, in Proceedings of the 22nd Particle Accelerator Conference, PAC-2007, Albuquerque, NM, edited by C. Petit-Jean Genaz (IEEE, New York, 2007), p. 3381, http://accelconf.web.cern.ch/AccelConf/p07/ PAPERS/THPAN070.PDF.

[15] W. Herr and F. Schmidt, A MAD-X primer, CERN AB Report No. CERN-AB-2004-027-ABP, 2004, https://cds .cern.ch/record/744163/files/ab-2004-027.pdf.

[16] http://mad.web.cern.ch/mad/.

[17] R. Cappi and M. Giovannozzi, Novel Method for Multiturn Extraction: Trapping Charged Particles in Islands of Phase Space, Phys. Rev. Lett. 88, 104801 (2002).

[18] R. Cappi and M. Giovannozzi, Multiturn extraction and injection by means of adiabatic capture in stable islands of phase space, Phys. Rev. ST Accel. Beams 7, 024001 (2004).

[19] S. Gilardoni, M. Giovannozzi, M. Martini, E. Métral, P. Scaramuzzi, R. Steerenberg, and A.-S. Müller, Experimental evidence of adiabatic splitting of charged particle beams using stable islands of transverse phase space, Phys. Rev. ST Accel. Beams 9, 104001 (2006).

[20] S. Gilardoni, M. Giovannozzi, M. Martini, E. Métral, P. Scaramuzzi, R. Steerenberg, and A.-S. Müller, Resonant multi-turn extraction: Principle and experiments, Nucl. Instrum. Methods Phys. Res., Sect. A 561, 249 (2006).

[21] M. Giovannozzi et al., Results from the 2009 beam commissioning of the CERN multi-turn extraction, in Proceedings of the International Particle Accelerator Conference, Kyoto, Japan, edited by A. Noda, Ch. Petit-Jean-Genaz, V. Schaa, T. Shirai, and A. Shirakawa (ICR, Kyoto, 2010), p. 3619, http://accelconf.web.cern.ch/ AccelConf/IPAC10/papers/thobmh02.pdf. 
[22] M. Giovannozzi and J. Morel, Principle and analysis of multiturn injection using stable islands of transverse phase space, Phys. Rev. ST Accel. Beams 10, 034001 (2007).

[23] A. Franchi, M. George, and M. Giovannozzi, First results of space charge simulations for the novel multi-turn injection, in Proceedings of the International Particle Accelerator Conference, Kyoto, Japan, edited by A. Noda, Ch. Petit-Jean-Genaz, V. Schaa, T. Shirai, and A. Shirakawa (ICR, Kyoto, 2010), p. 4710, http://accelconf .web.cern.ch/AccelConf/IPAC10/papers/thpe081.pdf.

[24] E. Courant and H. Snyder, Theory of the alternatinggradient synchrotron, Ann. Phys. (N.Y.) 3, 1 (1958).

[25] In the literature the tune is defined either with its integer part (6.25 for instance) or without ( 0.25 in our example). Results presented here are independent of this choice.

[26] R. Tomás García, Direct measurement of resonance driving terms in the super proton synchrotron (SPS) of CERN using beam position monitors, Ph.D. thesis, University of Valencia, 2003; Report No. CERN-THESIS-2003-010, https://cds.cern.ch/record/615164/files/thesis-2003-010.pdf.

[27] A. Franchi, R. Tomás, and F. Schmidt, Magnet strength measurement in circular accelerators from beam position monitor data, Phys. Rev. ST Accel. Beams 10, 074001 (2007).

[28] A. Bazzani, G. Servizi, E. Todesco, and G. Turchetti, A normal form approach to the theory of nonlinear betatronic motion, CERN Yellow Report No. 94-02, 1994, https://cds .cern.ch/record/262179/files/CERN-94-02.pdf.

[29] M. Giovannozzi, D. Quatraro, and G. Turchetti, Generating unstable resonances for extraction schemes based on transverse splitting, Phys. Rev. ST Accel. Beams 12, 024003 (2009).

[30] E. H. Poincaré, Sur un théorème de géométrie, Rend. Circ. Mat. Palermo 33, 375 (1912).

[31] G. D. Birkhoff, Proof of Poincaré's geometric theorem, Trans. Am. Math. Soc. 14, 14 (1913).

[32] G. D. Birkhoff, An extension of Poincaré's last geometric theorem, Acta Math. 47, 297 (1926).

[33] http://totem.web.cern.ch/Totem/.

[34] http://atlas-project-lumi-fphys.web.cern.ch/atlas-project -lumi-fphys/ALFA/.

[35] O. S. Brüning, P. Collier, P. Lebrun, S. Myers, R. Ostojic, J. Poole, and P. Proudlock, LHC design report Vol.1: The LHC main ring, LHC Report No. CERN-2004-003-V-1, 2004, https://cds.cern.ch/record/782076/files/CERN-2004003-V1.pdf. 